\title{
Using the rPatlak plot and dynamic FDG-PET to generate parametric images of relative local cerebral metabolic rate of glucose
}

\author{
WU YiGen ${ }^{1,2 *}$, ZHOU Yun ${ }^{3}$, BAO ShangLian ${ }^{2 *}$, HUANG SungCheng ${ }^{4}$, ZHAO XiaoHu $^{5}$ \& \\ LI Jun ${ }^{2}$ \\ ${ }^{1}$ School of Physics and Optoelectronic Engineering, Nanjing University of Information Science and Technology, Nanjing 210044, China; \\ ${ }^{2}$ Beijing City Key Laboratory of Medical Physics and Engineering, Peking University, Beijing 100871, China; \\ ${ }^{3}$ The Russell H. Morgan Department of Radiology and Radiological Science, School of Medicine, Johns Hopkins University, Baltimore, MD \\ 21287, USA; \\ ${ }^{4}$ Department of Molecular and Medical Pharmacology, UCLA David Geffen School of Medicine, University of California in Los Angeles, Los \\ Angeles, CA 90095, USA; \\ ${ }^{5}$ Imaging Department of Tongji Hospital of Tongji University, Shanghai 200065, China
}

Received June 28, 2011; accepted December 10, 2011

\begin{abstract}
Local cerebral metabolic rate of glucose (LCMRGlc) is an important index for the description of neural function. Dynamic ${ }^{18}$ F-fluoro-2-deoxy-D-glucose (FDG) positron emission tomography (PET) has been used for quantitative imaging of LCMRGlc in humans, but is seldom used routinely because of the difficulty in obtaining the input function noninvasively. A reference tissue-based Patlak plot model (rPatlak) was proposed to generate parametric images of LCMRGlc in a quantitative dynamic FDG-PET study without requiring blood sampling. Dynamic emission scans $(4 \times 0.5,4 \times 2$ and $10 \times 5$ min) were acquired simultaneously with an IV bolus injection of $155 \mathrm{MBq}$ of FDG. Arterial blood samples were collected during the scans via a catheter placed in the radial artery. Simulation data were also generated using the same scan sequence. The last ten scan data sets were used in a graphical analysis using the Patlak plot. The ratio of LCMRGlc estimated from the original Patlak (oPatlak, using plasma input) was used as the gold standard, and the standardized uptake value ratio (SUVR) was also calculated for comparison. Eight different tissues including white matter, gray matter, and whole brain were chosen as reference tissues for evaluation. Regardless of the reference region used, the slopes in the linear regression between oPatlak and rPatlak were closer to unity than the regression slopes between oPatlak and SUVR. The intercepts for the former were also closer to 0 than those for the latter case. The squared correlation coefficients were close to 1.0 for both cases. This showed that the results of rPatlak were in good agreement with those of oPatlak, however, SUVR exhibited more deviation. The simulation study also showed that the relative variance and bias for rPatlak were less than those for SUVR. The images obtained with rPatlak were very similar to those obtained with oPatlak, while there were differences in the relative spatial distribution between the images of SUVR and oPatlak. This study validates that the rPatlak method is better than the SUVR method and is a good approximation to the oPatlak method. The new method is suitable for generating LCMRGlc parametric images noninvasively.
\end{abstract}

quantification, local cerebral metabolic rate of glucose, FDG-PET, rPatlak method, SUVR, noninvasive parametric imaging

Citation: Wu Y G, Zhou Y, Bao S L, et al. Using the rPatlak plot and dynamic FDG-PET to generate parametric images of relative local cerebral metabolic rate of glucose. Chin Sci Bull, 2012, 57: 3811-3818, doi: 10.1007/s11434-012-5401-y

Local cerebral metabolic rate of glucose (LCMRGlc) is an important index to embody the neural function, ${ }^{18} \mathrm{~F}$-fluoro2-deoxy-D-glucose (FDG) with positron emission tomog-

*Corresponding authors (email: wuyge@126.com; bao@pku.edu.cn) raphy (PET) has been used for quantitative imaging of the local cerebral metabolic rate of glucose (LCMRGlc) in humans [1,2]. Usually, the plasma time activity curve (PTAC) is used as the input function, which is required in quantification of LCMRGlc with FDG-PET. The PTAC may be 
obtained by taking arterial or arterialized venous blood samples [1,3], but it is inconvenient for patients and involves many potential error sources, thus it is seldom used routinely. Some methods have been developed to minimize or eliminate the invasive blood sampling procedure in PET studies. Image-derived input function methods have been extensively investigated. These techniques have been used in cardiac studies [4-6], hepatic and renal PET studies [7,8] and brain studies $[9,10]$. However, these methods require corrections for the spillover from tissue-to-blood and the partial volume effects induced by the small size of the input function region of interest (ROI), and thus some venous blood samples are still needed. One study that evaluated image-derived input functions showed that without blood sampling the existing image-derived input function methods are not reliable for estimating LCMRGlc using dynamic FDG-PET [11]. To solve the problems inherent to image-derived input functions, an analytical PTAC model $[12,13]$ or a template calculated from a sample population $[14,15]$ were introduced, but these may also not match well with the special data of an idiographic subject.

Because of its short scan time (less than $15 \mathrm{~min}$ ) and avoidance of the need for blood sampling, the nonquantification method (tissue radioactivity) or the semiquantification method using standard uptake value (SUV) are still routinely used in generating LCMRGlc maps. The validity of SUV depends mainly on two important assumptions and approximations, one is that the non-sequestered FDG is a small fraction of the total radioactivity in tissue and can be neglected at late time T (e.g., larger than $45 \mathrm{~min}$ after FDG injection), and another is that the integral of the PTAC is proportional to the injected dose and inversely proportional to body weight or body surface area [16]. However, these two assumptions are not always valid, especially regarding the progression of disease. SUV and FDG flux estimated with the Patlak method $[17,18]$ may give opposite conclusions for some patients [19], and the discrepancies between these two indices are mainly affected by these two assumptions. In a comparison of tumor volumes [20], the values from the regional glucose metabolic rate (rGMR) maps (with Patlak analysis) were significantly smaller than the values from SUV maps, while the precise tumor volume is an important index for radiotherapy planning and therapy response monitoring. Consequently, the SUV reliability is somewhat controversial and its application as a quantitative index has been discouraged by some [21].

Previously, we proposed a new approach (rPatlak) [22] derived from the original Patlak (oPatlak) graphical approximation by using a reference tissue region chosen arbitrarily and without requiring any information from an input function for the quantification of LCMRGlc with FDG-PET. The approach requires neither arterial blood sampling nor corrections for spillover and partial volume effects that are required when using an image-derived input function. Some computer simulation studies have been carried out to evaluate this method, although the method has not yet been applied to human FDG-PET studies. Some papers have shown that SUVR provides equivalent accuracy to absolute SUV calculations normalized to dose and body weight [23] and is less variable than absolute measures of SUV [24]. Although SUVR is a semi-quantitative method, some studies [25,26] have shown that the finding with SUVR was dependent on the choice of reference regions. However, the rPatlak plot is a quantitative method and is independent on the choice of reference region. In this paper, we report the results of an application of the new method to a dynamic FDG-PET study of human subject. We also considered the selection of reference tissue and compared the results from the rPatlak and SUVR methods to the results from the oPatlak method (the gold standard) and generated parametric images of LCMRGlc with the rPatlak method.

\section{Materials and methods}

\subsection{The reference tissue-based Patlak plot model}

This method is derived from the original Patlak graphical approach, in which the relationship between tissue radioactivity concentration and arterial input function can be described by eq. (1):

$$
c_{\mathrm{i}}^{*}(t)=K \int_{0}^{t} c_{\mathrm{p}}^{*}(\tau) \mathrm{d} \tau+W c_{\mathrm{p}}^{*}(t), t>t_{0},
$$

where $c_{\mathrm{i}}^{*}(t)$ (the tissue time activity curve, i.e. TTAC) and $c_{\mathrm{p}}^{*}(t)$ (i.e. PTAC) denote the FDG activities at time $t$ in brain tissue and in arterial blood respectively, $K$ is the FDG influx constant from blood to tissue, $W$ is another constant related to the steady-state volume of the reversible compartments and effective plasma volume [17]. Both $c_{\mathrm{i}}^{*}(t)$ and $c_{\mathrm{p}}^{*}(t)$ are decay-corrected to the same time point (e.g., FDG injection time). Then, LCMRGlc can be calculated with $K$ using eq. (2) [27]:

$$
R=c_{\mathrm{p}} K / \mathrm{LC},
$$

where $c_{\mathrm{p}}$ is the glucose concentration in plasma, and LC denotes the lumped constant, which summarizes the difference between FDG and glucose in transportation and phosphorylation [27].

For two arbitrary regions, which are referred to as the reference region and the objective region in tissue, eq. (1) is applicable to both regions as shown in eqs. (3) and (4) below:

$$
\begin{aligned}
& c_{\mathrm{ir}}^{*}(t)=K_{\mathrm{r}} \int_{0}^{t} c_{\mathrm{p}}^{*}(\tau) \mathrm{d} \tau+W_{\mathrm{r}} c_{\mathrm{p}}^{*}(t), t>t_{0}, \\
& c_{\mathrm{io}}^{*}(t)=K_{\mathrm{o}} \int_{0}^{t} c_{\mathrm{p}}^{*}(\tau) \mathrm{d} \tau+W_{\mathrm{o}} c_{\mathrm{p}}^{*}(t), t>t_{0},
\end{aligned}
$$

where the subscript " $r$ " and "o" denote the reference region and the objective region respectively. It is assumed that the input function $c_{\mathrm{p}}^{*}(t)$ is the same for both regions. From eqs. 
(3) and (4) the equation below (eq. (5)) [22] can be derived,

$$
\begin{aligned}
\int_{t_{0}}^{t} c_{\mathrm{io}}^{*}(\tau) \mathrm{d} \tau= & r K \int_{t_{0}}^{t} c_{\mathrm{ir}}^{*}(\tau) \mathrm{d} \tau+W_{\mathrm{or}}\left(c_{\mathrm{ir}}^{*}(t)\right. \\
& \left.-c_{\mathrm{ir}}^{*}\left(t_{0}\right)\right)+W_{\mathrm{rr}}\left(c_{\mathrm{io}}^{*}\left(t_{0}\right)-c_{\mathrm{io}}^{*}(t)\right)
\end{aligned}
$$

where $r K=K_{\mathrm{o}} / K_{\mathrm{r}}, \quad W_{\mathrm{rr}}=W_{\mathrm{r}} / K_{\mathrm{r}}, \quad W_{\mathrm{or}}=W_{\mathrm{o}} / K_{\mathrm{r}}, t_{0}$ (about $10 \mathrm{~min}$ ) is the time of the first PET scan used for Patlak analysis. In eq. (5), the arterial input function $c_{\mathrm{p}}^{*}(t)$ has already been eliminated, so the relative FDG flux $(r K)$ in the objective region to a reference region can be obtained noninvasively. Because the values of $c_{\mathrm{p}}$ and LC are the same for all regions, the relative LCMRGlc in the objective region to a reference region is also equal to $r K$, based on eq. (2). Thus by using eq. (5), the relative LCMRGlc (rLCMRGlc) can be estimated directly with the linear least squares method (see details in [22]). Then, absolute LCMRGlc can also be calculated with $r K$ using eq. (6) [22] instead of eq. (2),

$$
R=R_{\mathrm{g}} \times r K_{\mathrm{g}},
$$

where $r K_{\mathrm{g}}$ is just the relative FDG flux in a local area (or a voxel) to the whole brain and can easily be estimated with the present method, $R_{\mathrm{g}}$ is a constant parameter for the average metabolic rate of the whole brain. This is the rPatlak method.

Since the rPatlak method does not estimate $K$ but only $r K$ relative to the reference region, an analogous $r K$ is also calculated using oPatlak and SUVR methods for comparison with the same two regions. Using eqs. (3) and (4), the $K$ value of the objective region and the reference region can be fitted individually, so that the value of $r K$ obtained with the oPatlak method is the ratio of these two values $\left(K_{\mathrm{o}} / K_{\mathrm{r}}\right)$. For the SUVR method, we simply denote $r K=c_{\mathrm{io}}^{*}(t) / c_{\mathrm{ir}}^{*}(t)$.

\subsection{Computer simulations}

In this study, computer simulations including TTAC model and random parameters are similar to those described in [22]. The main differences are: (1) A PTAC from a human FDG study was used as the input function. (2) Three sets of typical transport rate constants obtained in a human study [3] were used to generate the TTACs of the reference regions. They are $\left(k_{1}{ }^{*}, k_{2}{ }^{*}, k_{3}{ }^{*}, k_{4}{ }^{*}\right)=(0.1020,0.1300,0.0620$, $0.0068),(0.0540,0.1090,0.0450,0.0058)$ and $(0.0761$, $0.1187,0.0528,0.0063) \mathrm{min}^{-1}$ and are denoted as grey matter (GM), white matter (WM) and whole brain (WB) respectively. (3) Gaussian noise was not added to the TTAC of the reference region. The reason is that the reference region may consist of many voxels, so the noise level of its TTAC is very small. (4) The decay correction factor $\exp \left(0.693 t_{j}^{\prime} / \lambda\right)$ was added to the TTAC's error variance calculation. The formula used for the simulated noise is $\sigma^{2}\left(t_{j}^{\prime}\right)=\alpha \times \bar{c}_{i}^{*}\left(t_{j}^{\prime}\right) \exp \left(0.693 t_{j}^{\prime} / \lambda\right) / \delta t_{j} \quad[28,29]$, where $\alpha$ is the noise level and was set to 0 (noise free), 0.1, 0.5, 1.0, 2.0, and 4.0, $\bar{c}_{i}^{*}\left(t_{j}^{\prime}\right)=\int_{t_{j-1}}^{t_{j}} c_{i}(\tau) \mathrm{d} \tau / \delta t_{j}$ is the average value of $c_{\mathrm{i}}^{*}(t)$ over the length of the $j$ th scan interval, $t_{j}^{\prime}=\left(t_{j-1}+t_{j}\right) / 2$ is the mid-time of the $j$ th scan, $\delta t_{j}=t_{j-1}-t_{j}$ is the length of the $j$ th scan interval, and $\lambda$ is the physical half-life of F-18 (110 min).

\subsection{Application to human FDG-PET studies}

A set of dynamic FDG-PET data was acquired from a normal, healthy human volunteer using an ECAT EXACT HR+ PET scanner (axial field of view $=15.5 \mathrm{~cm}$; intrinsic full-width-at-half-maximum (FWHM) at the center $=4.3$ $\mathrm{mm}$ ) in 3-D acquisition mode. The study was approved by the UCLA Human Subject Protection committee and was performed in the 1990s. A transmission scan was carried out using a ${ }^{68} \mathrm{Ge}$ line source for attenuation correction prior to intravenous FDG administration. Then, dynamic emission scans using a sequence of $4 \times 0.5,4 \times 2$ and $10 \times 5 \mathrm{~min}$, for a total 60 min (18 frames) were started simultaneously with an intravenous bolus injection of $155 \mathrm{MBq}$ of FDG. For each PET scan frame, 63 transaxial images $(128 \times 128$ pixel; $1.446 \mathrm{~mm}$ pixel size; $2.425 \mathrm{~mm}$ plane thickness) were reconstructed using a filtered back-projection algorithm with a Hanning filter (cut-off frequency of 0.3 cycle per projection element), resulting in an in-plane spatial resolution of $\sim 8 \mathrm{~mm}$ FWHM. Simultaneously, dead time, scatter, and measured attenuation corrections were also applied. PTAC was obtained by collecting 25 serial arterial blood samples via a catheter in the radial artery during the dynamic scans. The data of last ten frames (from 10 to $60 \mathrm{~min}$ ) were used in rPatlak and oPatlak analysis (this means that $t_{0}=12.5 \mathrm{~min}$ ), while the data of last frame (55-60 min) were used for the SUVR calculation. The arterial blood samples were used only in the oPatlak analysis.

\subsection{Selection of reference region for human data}

Eight different regions were also chosen as reference regions in the analysis of the human study data. These regions were $\mathrm{WM}, \mathrm{GM}, \mathrm{WM}+\mathrm{GM}$ (W\&GM), voxels within $20 \%$, $25 \%, 28 \%, 30 \%$ of the maximum image activity (M20, M25, M28, M30) of the last PET scan and WB (60\% GM + 40\% WM) respectively. M25 was defined as the whole brain as with the "whole-slice" described in [30]. The 60\% GM + $40 \% \mathrm{WM}$ was also defined as the whole brain as in [31]. The white matter, grey matter and other regions were segmented automatically by clustering the dynamic 3D FDG-PET data with the method used previously [32], which combines the K-means cluster analysis method [33] and the average linkage hierarchical clustering method [34]. The segmentation procedures are as follows: (a) Obtaining a 
mask image of the brain by averaging all frames of the time series and smoothing with a 6-mm FWHM Gaussian filter using SPM2 (http://www.fil.ion.ucl.ac.uk/spm) (That means the intensity for all voxels outside of the head were masked to zero in all PET dynamic images.); (b) applying 3D smoothing to the masked PET 3D data of each frame; (c) classifying voxel TTACs of the masked and smoothed PET $3 \mathrm{D}$ dynamic data into 15 clusters with the K-means method; (d) classifying the 15 averaged TTACs from the 15 clusters into 4 final clusters labelled white matter, gray matter, scalp and vasculature with the hierarchical method. The smoothing was only used in the clustering procedure and was not used in the subsequent analyses.

In the regression analyses, the voxels in W\&GM were also chosen as the objective region, but in generating maps, all voxels were chosen as the objective region. To compare results of the three calculations of $r K$, the linear regression $y$ $=S x+I$ was carried out using an unweighted least squares method, where $x, y$ are two $r K$ values calculated with oPatlak and rPatlak (or SUVR), and $S$ and $I$ are the slope and intercept in the regression, respectively. In the computer simulation study, the relative root mean square error (RMSE\%) and the relative bias (Bias\%) were used to assess the accuracy of the three calculations of $r K$. They are defined as:

$$
\begin{aligned}
\operatorname{RMSE}(\%) & =\sqrt{\sum_{1}^{n}\left(p_{i}^{\mathrm{cal}} / p_{i}^{\text {true }}-1\right)^{2} /(n-1)} \times 100, \\
\operatorname{Bias}(\%) & =\sum_{1}^{n}\left(p_{i}^{\text {cal }} / p_{i}^{\text {true }}-1\right) / n \times 100,
\end{aligned}
$$

where $p_{i}^{\text {true }}$ is the $r K$ true value (from the noise free TTAC) of the $i$ th simulation event calculated with the oPatlak method; $p_{i}^{\text {cal }}$ is the $r K$ real value (from the noise TTAC) of the $i$ th event calculated with the oPatlak, rPatlak and SUVR method. To quantify the differences between two images in human studies, the root mean square relative difference (RMSRD) between image 1 and 2 was used, where $\operatorname{RMSRD}=\sqrt{\sum_{1}^{n}\left(p_{i}^{1} / p_{i}^{2}-1\right)^{2} / n}, p_{i}{ }^{1}$ and $p_{i}{ }^{2}$ are the intensities of the $i$ th voxel in image 1 and 2 respectively.

\section{Results}

Because the $r K$ value depends upon the reference region selection, if the intercept $I$ is not equal to zero, it will also depend on the reference region selection. To avoid this dependence, we also performed another regression using all $r K$ values corrected to those with the reference region M25 (the whole brain). After directly calculating $r K$ with the rPatlak method using a selected reference region, all values were corrected by multiplying the ratio of the two absolute FDG fluxes calculated with the oPatlak method in the selected reference region and in M25. Note that the two slopes and the squared correlation coefficients $\left(R^{2}\right)$ should be equal in the two regressions when using uncorrected and corrected $r K$ values, while the two intercepts, denoted as $I_{1}$ and $I_{2}$, may not be equal.

Figures 1 and 2 show two examples of the correlation between two $r K$ values calculated with the oPatlak method and the rPatlak (or SUVR) method for the simulation study using $\mathrm{WB}$ as the reference region under noise level $=1.0$ and for the human PET study using M25 as the reference
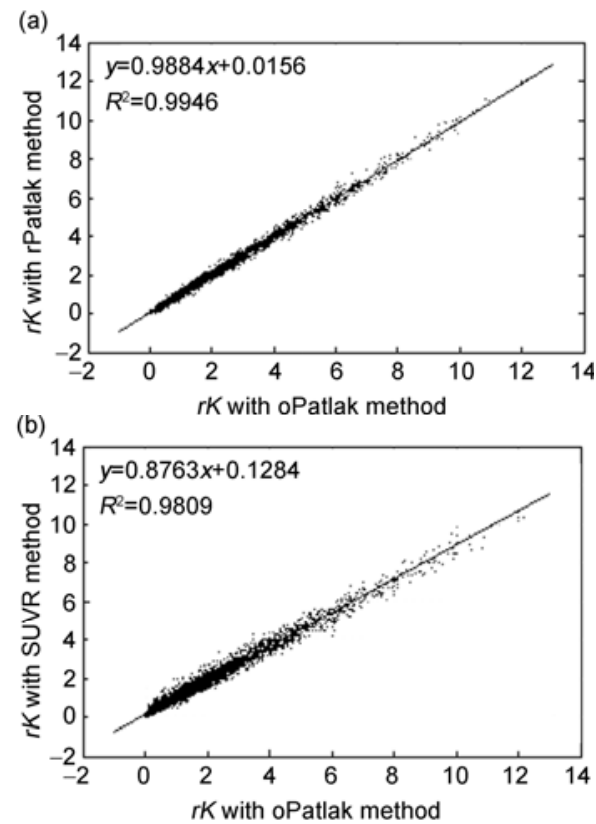

Figure 1 Correlation of $r K$ calculated with the oPatlak method (abscissa) and the rPatlatk (a) or SUVR (b) method (ordinate) using WB as the reference region under a noise level $=1.0$ for the simulation study.
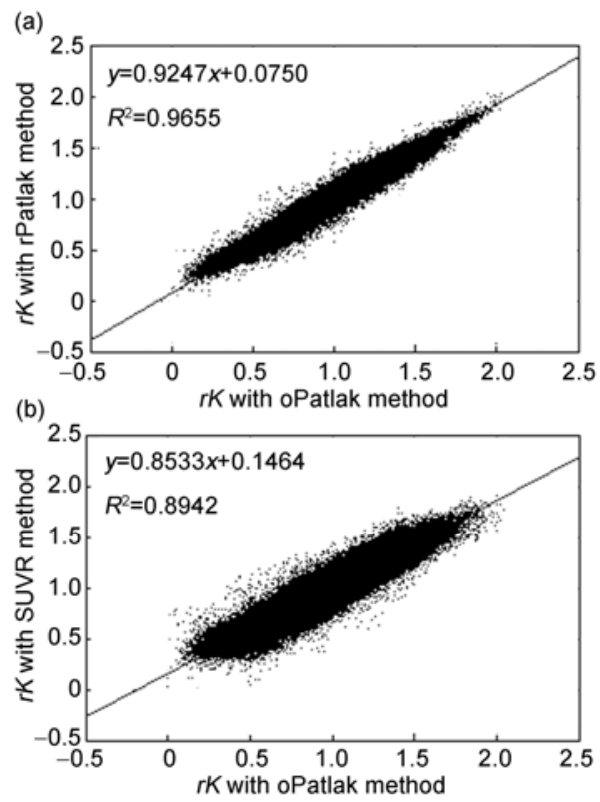

Figure 2 Correlation of $r K$ calculated with the oPatlak method (abscissa) and the rPatlatk (a) or SUVR (b) method (ordinate) using M25 as the reference region for the human PET study. 
region. Tables 1 and 2 give the values of the regression parameters for the simulation and the human study, respectively. We found that for both cases, regardless of the reference region used, the slopes in the linear regression between the oPatlak and rPatlak methods are closer to unity than those in the regression between oPatlak and SUVR. The intercepts for the former case are also closer to 0 than those for the latter case and the $R^{2}$ value is close to 1 for both. These indicate that rPatlak is in good agreement with oPatlak, while SUVR is in somewhat less good agreement. From these tables, we also found that the slopes and intercepts (corrected) varied little with the selected reference regions. This indicated that the rPatlak method did not depend upon the reference region selection.

Figure 3(a) shows that up to very high noise level ( $\alpha=$ $4.0)$, the Bias\% with oPatlak is very small (close to zero), the Bias\% with rPatlak is less than 2 and the Bias\% with SUVR is less than 6 , but was more than that with rPatlak.
Figure 3(b) shows that under low noise level $(<1)$, the RMSE\% with oPatlak and rPatlak were almost equal but much less than that with SUVR. Under a high noise level, the RMSE\% with rPatlak is greater than that with oPatlak but was still less than that with SUVR.

Using M25 as the reference region, three rLCMRGlc parametric images were generated with the rPatlak, oPatlak and SUVR methods, and are shown in Figure 4. The image obtained with rPatlak is very similar to that obtained with oPatlak, while there were differences between the images obtained with SUVR and oPatlak. The RMSRD for the images obtained with rPatlak and oPatlak was $9.1 \%$, while that for images obtained with SUVR and oPatlak was $21.1 \%$. These results show that the rPatlak method is a good approximation to the oPatlak method and is better than the SUVR method.

The $t_{0}$ selection is another problem on the oPatlak and rPatlak methods. In most dynamic FDG-PET studies, $t_{0}$ was

Table 1 Voxel-wise comparison of $r K$ with oPatlak and rPatlak (or SUVR) method (simulation study)

\begin{tabular}{|c|c|c|c|c|c|c|c|}
\hline \multirow{2}{*}{ Noise level } & \multirow{2}{*}{ Reference region } & \multicolumn{3}{|c|}{ rPatlak } & \multicolumn{3}{|c|}{ SUV } \\
\hline & & Slope & Intercept & $R^{2}$ & Slope & Intercept & $R^{2}$ \\
\hline \multirow[t]{3}{*}{0.0} & GM & 0.9658 & -0.0055 & 0.9961 & 0.8932 & 0.0927 & 0.9940 \\
\hline & WM & 0.9489 & -0.0319 & 0.9890 & 0.8578 & 0.1810 & 0.9940 \\
\hline & WB & 0.9590 & -0.0191 & 0.9939 & 0.8760 & 0.1263 & 0.9940 \\
\hline \multirow[t]{2}{*}{0.1} & GM & 0.9822 & 0.0174 & 0.9992 & 0.8940 & 0.0923 & 0.9926 \\
\hline & WM & 0.9865 & 0.0263 & 0.9991 & 0.8585 & 0.1802 & 0.9926 \\
\hline \multirow[t]{3}{*}{0.5} & GM & 0.9837 & 0.0161 & 0.9972 & 0.8941 & 0.0930 & 0.9873 \\
\hline & WM & 0.9882 & 0.0235 & 0.9971 & 0.8586 & 0.1816 & 0.9873 \\
\hline & WB & 0.9882 & 0.0160 & 0.9971 & 0.8768 & 0.1267 & 0.9873 \\
\hline \multirow[t]{3}{*}{1.0} & GM & 0.9839 & 0.0160 & 0.9948 & 0.8936 & 0.0942 & 0.9809 \\
\hline & WM & 0.9884 & 0.0231 & 0.9946 & 0.8581 & 0.1840 & 0.9809 \\
\hline & WB & 0.9884 & 0.0156 & 0.9946 & 0.8763 & 0.1284 & 0.9809 \\
\hline \multirow[t]{2}{*}{2.0} & GM & 0.9836 & 0.0163 & 0.9900 & 0.8919 & 0.0973 & 0.9684 \\
\hline & WB & 0.9883 & 0.0160 & 0.9897 & 0.8747 & 0.1325 & 0.9684 \\
\hline \multirow[t]{3}{*}{4.0} & GM & 0.9825 & 0.0181 & 0.9807 & 0.8870 & 0.1063 & 0.9445 \\
\hline & WM & 0.9873 & 0.0271 & 0.9802 & 0.8518 & 0.2076 & 0.9445 \\
\hline & WB & 0.9875 & 0.0181 & 0.9801 & 0.8699 & 0.1448 & 0.9445 \\
\hline
\end{tabular}

Table 2 Voxel-wise comparison of $r K$ with oPatlak and rPatlak (or SUVR) method (human PET study) ${ }^{\text {a) }}$

\begin{tabular}{|c|c|c|c|c|c|c|c|c|}
\hline \multirow{2}{*}{ Reference region } & \multicolumn{4}{|c|}{ rPatlak } & \multicolumn{4}{|c|}{ SUV } \\
\hline & Slope & $I_{1}$ & $I_{2}$ & $R^{2}$ & Slope & $I_{1}$ & $I_{2}$ & $R^{2}$ \\
\hline WM & 0.9065 & 0.1074 & 0.0792 & 0.9642 & 0.8297 & 0.1931 & 0.1424 & 0.8942 \\
\hline GM & 0.9227 & 0.0673 & 0.0792 & 0.9648 & 0.8579 & 0.1251 & 0.1472 & 0.8942 \\
\hline W\&GM & 0.9165 & 0.0836 & 0.0787 & 0.9647 & 0.8459 & 0.1541 & 0.1451 & 0.8942 \\
\hline M20 & 0.9273 & 0.0692 & 0.0749 & 0.9655 & 0.8581 & 0.1360 & 0.1473 & 0.8942 \\
\hline M25 & 0.9247 & 0.0750 & 0.0750 & 0.9655 & 0.8533 & 0.1464 & 0.1464 & 0.8942 \\
\hline M30 & 0.9233 & 0.0777 & 0.0707 & 0.9662 & 0.8431 & 0.1590 & 0.1446 & 0.8942 \\
\hline WB & 0.9184 & 0.0787 & 0.0788 & 0.9647 & 0.8494 & 0.1456 & 0.1457 & 0.8942 \\
\hline
\end{tabular}

a) $I_{1}$, intercept fitted from the uncorrected $r K$ values; $I_{2}$, intercept fitted from the corrected $r K$ values. 

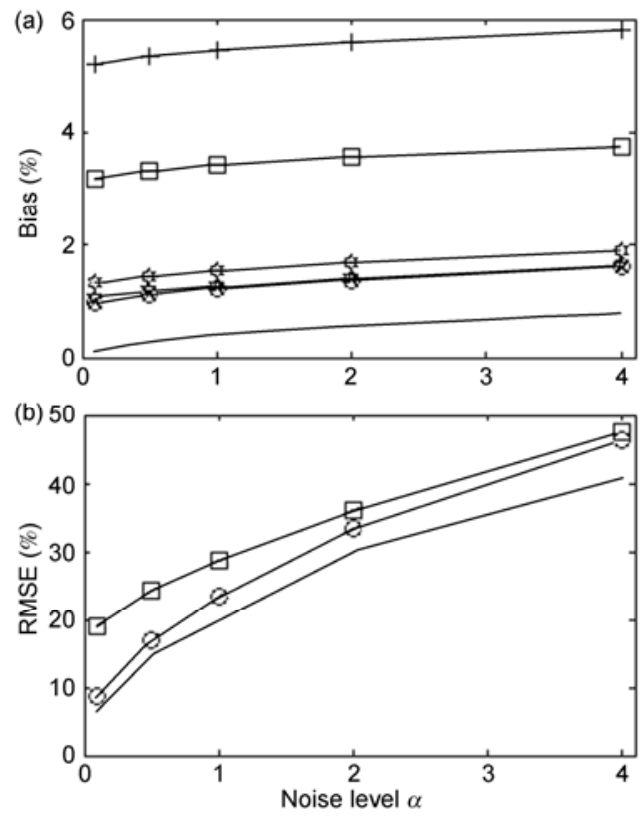

Figure 3 Relative bias (a) and RMSE (b) change with noise level. - , oPatlak method; rPatlak method, GM as the reference region; $\odot$, rPatak method, WM as the reference region; $\rightarrow$, rPatlak method, WB as the reference region; + , SUVR method, GM as the reference region; , SUVR method, WM as the reference region; $\square$, SUVR method, $\mathrm{WB}$ as the reference region.

chosen to be from 10 to $30 \mathrm{~min}$. We also used computer simulations and human data to make a comparison of $r K$ values obtained with the rPatlak and oPatlak methods by selecting $t_{0}=12.5,17.5$ and $22.5 \mathrm{~min}$. The results are given in Table 3. For the computer simulation study, WB was chosen as the reference region under noise level $=1$. In the human study, M25 was chosen as the reference region. For both the computer simulation and the human study, the correlations between the $r K$ values obtained with the rPatlak and oPatlak methods using three different $t_{0}$ were nearly equal. For both the oPatlak and the rPatlak methods, the slopes in linear regression for different $t_{0}$ were very close to unity. Furthermore, the intercepts were very close to 0 and the $R^{2}$ were close to 1 . These results indicate that in the range from 12.5 to $22.5 \mathrm{~min}$ the $t_{0}$ effect on $r K$ values obtained with oPatlak and rPatlak method was not large. Obviously, the RMSE\% of $r K$ will increase with the $t_{0}$ increase, for both oPatlak and rPatlak methods. The RMSE\% values were from $23.45 \%$ to $29.91 \%$ for the rPatlak method and were from $20.43 \%$ to $28.93 \%$ for the oPatlak method when $t_{0}$ was from 12.5 to $22.5 \mathrm{~min}$.

\section{Discussion}

This study validated that the rPatlak method can be used to estimate the relative FDG flux $r K$ as a good approximation to the oPatlak approach. However, without other constraints,

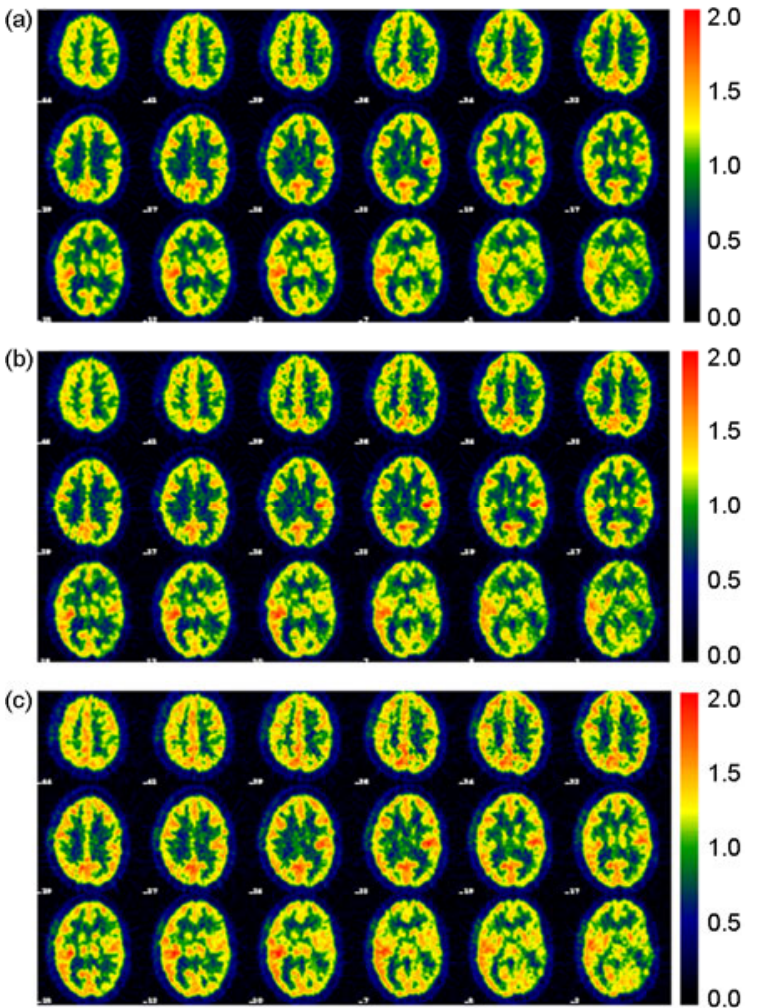

Figure $4 \quad r K$ maps calculated with the rPatlak (a), oPatlatk (b) and SUVR (c) method using M25 as the reference region.

the absolute $K$ value cannot be calculated with the rPatlak method. This is the main weakness of this kind of reference region method. Because the choice of tissue region that is used as the reference region is not important for the rPatlak method, a simple approach is to select the whole brain (e.g., M25) as the reference region. For this case, eq. (6) may be used to calculate the absolute LCMRGlc value instead of eq. (2). Eqs. (2) and (6) are two methods to calculate the absolute LCMRGlc. Eq. (2) requires the constant parameter LC and the local absolute FDG flux $K$; eq. (6) requires the constant parameter $R_{\mathrm{g}}$ and the local relative FDG flux $r K_{\mathrm{g}}$ in a local area to the whole brain. Calculating $K$ with other methods requires the input function, but calculating $r K_{\mathrm{g}}$ with rPatlak method does not.

For those situations that require an absolute LCMRGlc value, one can add a constant like LC or $R_{\mathrm{g}}$ to calibrate the relative value. In the present study, absolute LCMRGlc values in grey matter and white matter were calculated with the rPatlak method using eq. (6) and with the oPatlak method using eqs. (2) and (6). The results are given in Table 4 , where $R_{\mathrm{g}}$ and LC were taken from [3]. The relative differences between the absolute LCMRGlc values obtained using eq. (6) with the rPatlak and oPatlak methods were less than $2 \%$. The relative differences between the absolute LCMRGlc values obtained using rPatlak (eq. (6)) and the values with oPatlak method (eq. (2)) (a traditional method that requires the input function) were approximately $25 \%$. 
Table 3 Voxel-wise comparison of $r K$ using three different $t_{0}{ }^{\text {a) }}$

\begin{tabular}{|c|c|c|c|c|c|c|}
\hline \multirow{2}{*}{ Regressed variates } & \multicolumn{3}{|c|}{ Simulation data (noise level = 1) } & \multicolumn{3}{|c|}{ Human PET data } \\
\hline & Slope & $I$ & $R^{2}$ & Slope & $I$ & $R^{2}$ \\
\hline$r K_{r 1}$ vs. $r K_{o 1}$ & 0.9884 & 0.0156 & 0.9946 & 0.9310 & 0.0669 & 0.9911 \\
\hline$r K_{r 2}$ vs. $r K_{o 2}$ & 0.9593 & 0.0638 & 0.9927 & 0.9179 & 0.0811 & 0.9526 \\
\hline$r K_{r 3}$ vs. $r K_{o 3}$ & 0.9556 & 0.0537 & 0.9883 & 0.9062 & 0.0917 & 0.9278 \\
\hline$r K_{r 2}$ vs. $r K_{r 1}$ & 0.9759 & 0.0190 & 0.9974 & 1.0120 & 0.0125 & 0.9740 \\
\hline$r K_{r 3}$ vs. $r K_{r 1}$ & 0.9703 & 0.0141 & 0.9881 & 0.9987 & -0.0030 & 0.9194 \\
\hline$r K_{r 3}$ vs. $r K_{r 2}$ & 0.9958 & 0.0075 & 0.9936 & 1.0249 & 0.0001 & 0.9612 \\
\hline$r K_{o 3}$ Vs. $r K_{o 1}$ & 0.9857 & -0.0004 & 0.9896 & 0.9721 & -0.0030 & 0.9194 \\
\hline$r K_{o 3}$ vs. $r K_{o 2}$ & 0.9946 & -0.0002 & 0.9948 & 0.9903 & 0.0043 & 0.9545 \\
\hline
\end{tabular}

a) $r K_{r i}$ and $r K_{o i}(i=1,2,3), r K$ values with rPatlak and oPatlak method using $t_{0}=12.5,17.5$ and 22.5 min respectively. For the comparisons, WB and M25 were chosen as the reference regions for the simulation study and the human PET study respectively.

Table 4 Absolute LCMRGlc values (in unit of $\mu \mathrm{mol} /(100 \mathrm{~g} \mathrm{~min}$ ) computed by three methods and their relative differences (human PET study) ${ }^{\text {a) }}$

\begin{tabular}{cccccc}
\hline Tissue & Method 1 & Method 2 & Method 3 & D1,2(\%) & D1,3(\%) \\
\hline WM & $22.33 \pm 3.20$ & $22.03 \pm 3.15$ & $17.71 \pm 3.87$ & 1.36 & 26.09 \\
GM & $35.10 \pm 5.02$ & $35.17 \pm 5.03$ & $28.22 \pm 4.90$ & -0.20 & 24.38 \\
\hline
\end{tabular}

a) Method 1, rPatlak using eq. (6); Method 2, oPatlak using eq. (6); Method 3, oPatlak using eq. (2). D1,2(\%) $=($ LCMRGlc value with Method 1/LCMRGlc value with Method $2-1.0) \times 100 ;$ D1,3(\%) $=($ LCMRGlc value with Method 1/LCMRGlc value with Method $3-1.0) \times 100$.

As the absolute LCMRGlc value is an important physiological parameter, more investigations are warranted to obtain this parameter non-invasively and reliably.

Using an arbitrary brain tissue region, the quantified rLCMRGlc $(r K)$ image can be generated automatically, noninvasively and quickly by the rPatlak method. Because the reliability of SUV is still somewhat controversial [21], the use of the rPatlak method can be considered instead. Usually, the relative distribution of LCMRGlc can indicate the physiologic or pathologic states and relative LCMRGlc values have a smaller variability than absolute values $[24,35]$. In the functional study to assess changes between two different metabolic states of a single subject, we may automatically select those voxels that are within $25 \%$ of the maximum image activity (M25, one of the definitions for whole brain) as the reference region to generate the relative LCMRGlc image. The scaling or AnCova model [36] may also be used to remove the global effect.

\section{Conclusions}

This study has validated that the relative Paklak method is more robust than the SUVR method and is a good approximation to the original Patlak method. Although the total scan time (greater than $30 \mathrm{~min}$ ) with the rPatlak method is longer than that with the SUVR method (less than $15 \mathrm{~min}$ ), this may be the cost of obtaining accurate quantified parameters. As with the SUVR approach, the new method does not require an input function. With the rPatlak method, the reference region may be selected arbitrarily and the calcula- tion is straightforward; thus, it may be suitable for the routine generation of rLCMRGlc parametric images.

The authors would like to thank Dr. Hung XinRui for her assistance in pre-processing the data. This work was supported by the National Natural Science Foundation of China (30840033, 30770615 and 30970818), the National Basic Research Program of China (2011CB707701) and the Joint Research Foundation of Beijing Education Committee (JD100010607).

1 Phelps M, Huang S C, Hoffman E, et al. Tomographic measurement of local cerebral glucose metabolic rate in humans with F-18-2fluoro-2-deoxy-D-glucose: Validation of method. Ann Neurol, 1979, 6: $371-388$

2 Reivich M, Kuhl D, Wolf A J, et al. The 18-F-fluorodeoxyglucose method for the measurement of local cerebral glucose metabolism in man. Circ Res, 1979, 44: 127-137

3 Huang S C, Phelps M, Hoffman E, et al. Noninvasive determination of local cerebral metabolic rate of glucose in man. Amer J Physiol, 1980, 238: E69-E82

4 Weinberg I N, Huang S C, Hoffman E J, et al. Validation of PET-acquired input functions for cardiac studies. J Nucl Med, 1988, 29: 241-247

5 Gambhir S S, Schwaiger M, Huang S C, et al. Simple noninvasive quantification method for measuring myocardial glucose utilization in humans employing positron emission tomography and fluorine-18 deoxyglucose. J Nucl Med, 1989, 30: 359-366

6 Iida $\mathrm{H}$, Rhodes $\mathrm{C} \mathrm{G}$, de Silva R, et al. Use of the left ventricular time activity curve as a noninvasive input function in dynamic oxygen-15 water positron emission tomography. J Nucl Med, 1992, 33: 16691677

7 Germano G, Chen B C, Huang S C, et al. Use of the abdominal aorta for arterial input function determination in hepatic and renal PET studies. J Nucl Med, 1992, 33: 613-620

8 Chen B C, Germano G, Huang S C, et al. A new noninvasive quantification of renal blood flow with $\mathrm{N}-13$ ammonia, dynamic positron emission tomography, and a two-compartment model. J Am Soc Nephrol, 1992, 3: 1295-1306

9 Chen K W, Bandy D, Reiman E, et al. Noninvasive quantification of the cerebral metabolic rate for glucose using positron emission tomography, ${ }^{18}$ F-fluoro-2-deoxyglucose, the Patlak method, and an image-derived input function. J Cereb Blood Flow Metab, 1998, 18: 716-723

10 Parker B, Feng D. Graph-based Mumford-Shah segmentation of dynamic PET with application to input function estimation. IEEE Trans Nucl Sci, 2005, 52: 79-89

11 Zanotti-Fregonara P, Fadaili E M, Maroy R, et al. Comparison of eight methods for the estimation of the image-derived input function in dynamic $\left[{ }^{18} \mathrm{~F}\right]$-FDG PET human brain studies. J Cereb Blood Flow Metab, 2009, 29: 1825-1835 
12 Feng D, Huang S C, Wang X. Models for computer simulation studies of input functions for tracer kinetic modeling with positron emission tomography. Int J Biomed Comput, 1993, 32: 95-110

13 Phillips R L, Chen C Y, Wong D F, et al. An improved method to calculate cerebral metabolic rates of glucose using PET. J Nucl Med, 1995, 36: 1668-1679

14 Takikawa S, Dhawan V, Spetsieris P, et al. Noninvasive quantitative fluorodeoxyglucose PET studies with an estimated input function derived from a population-based arterial blood curve. Radiology, 1993, 188: $131-136$

15 Sundaram S K, Freedman N M T, Carrasquillo J A, et al. Simplified kinetic analysis of tumor 18F-FDG uptake: A dynamic approach. J Nucl Med, 2004, 45: 1328-1333

16 Huang S C. Anatomy of SUV. Nucl Med Biol, 2000, 27: 643-646

17 Patlak C S, Blasberg R, Fenstermacher J D. Graphical evaluation of blood to brain transfer constants from multiple-time uptake data. J Cereb Blood Flow Metab, 1983, 3: 1-7

18 Patlak C S, Blasberg R G. Graphical evaluation of blood to brain transfer constants from multiple-time uptake data: Generalizations. J Cereb Blood Flow Metab, 1985, 5: 584-590

19 Freedman N M T, Sundaram S K, Kurdziel K, et al. Comparison of SUV and Patlak slope for monitoring of cancer therapy using serial PET scans. Eur J Nucl Med, 2003, 30: 46-53

20 Visser E P, Philippens M E P, Kienhorst L, et al. Comparison of tumor volumes derived from glucose metabolic rate maps and SUV maps in dynamic ${ }^{18}$ F-FDG PET. J Nucl Med, 2008, 49: 892-898

21 Keyes J W J. SUV: Standard uptake or silly useless value? J Nucl Med, 1995, 36: 1836-1839

22 Wu Y G. Noninvasive quantification of local cerebral metabolic rate of glucose for clinical application using positron emission tomography and ${ }^{18}$ F-fluoro-2-deoxy-D-glucose. J Cereb Blood Flow Metab, 2008, 28: 242-250

23 Lowe V J, Kemp B J, Jack C R, et al. Comparison of 18F-FDG and PiB PET in cognitive impairment. J Nucl Med, 2009, 50: 878-886

24 Wang G J, Volkow N D, Wolf A P, et al. Intersubject variability of brain glucose metabolic measurements in young normal males. J Nucl Med, 1994, 35: 1457-1466

25 Yakushev I, Landvogt C, Buchholz H G, et al. Choice of reference area in studies of Alzheimer's disease using positron emission tomography with fluorodeoxyglucose-F18. Psychiatry Res Neuroimaging, 2008, 164: 143-153
26 Chen K W, Langbaum J, Fleisher A, et al. Twelvemonth metabolic declines in probable Alzheimer's disease and amnestic mild cognitive impairment assessed using an empirically pre-defined statistical region-of-interest: Findings from the Alzheimer's disease neuroimaging initiative. NeuroImage, 2010, 51: 654-664

27 Sokoloff L, Reivich M, Kennedy C, et al. The C-14-deoxyglucose method for the measurement of local cerebral glucose utilization: Theory, procedure, and normal values in the conscious and anesthetized albino rat. J Neurochem, 1977, 28: 897-916

28 Chen K W, Huang S C, Yu D C. The effects of measurement errors in the plasma radioactivity curve on parameter estimation in positron emission tomography. Phys Med Biol, 1991, 36: 1183-1200

29 Feng D, Wong K P, Wu C M, et al. A technique for extracting physiological parameters and the required input function simultaneously from PET image measurements: Theory and simulation study. IEEE Trans Inform Technol Biomed, 1997, 1: 243-254

30 Hasselbalch S G, Madsen P L, Knudsen G M, et al. Calculation of the FDG lumped constant by simultaneous measurements of global glucose and FDG metabolism in humans. J Cereb Blood Flow Metab, 1998, 8: 154-160

31 Reivich M, Alavi A, Wolf A, et al. Glucose metabolic rate kinetic model parameter determination in humans: The lumped constants and rate constants for F-18-fluorodeoxyglucose and C-11-deoxyglucose. J Cereb Blood Flow Metab, 1985, 5: 179-192

32 Huang X R, Zhou Y, Bao S L, et al. Clustering based linear least square fitting method for generation of parametric images in dynamic FDG PET studies. Int J Biomed Imaging, 2007, Article ID 65641, doi:10.1155/2007/65641

33 Wong K P, Feng D G, Meikle S R. Segmentation of dynamic PET images using cluster analysis. IEEE Trans Nucl Sci, 2002, 49: 200-207

34 Zhou Y, Huang S C, Bergsneider M, et al. Improved parametric image generation using spatial-temporal analysis of dynamic PET studies. NeuroImage, 2002, 15: 697-707

35 Minoshima S, Frey K A, Foster N L, et al. Preserved pontine glucose metabolism in Alzheimer disease: A reference region for functional brain image (PET) analysis. J Comput Assist Tomogr, 1995, 19: 541-547

36 Kiebel S, Homes A. The General Linear Model. In: Frackowiak R, Friston K, Frith C, et al., eds. Human Brain Function. 2nd ed. San Diego, CA: Elsevier, 2004. 15-16

Open Access This article is distributed under the terms of the Creative Commons Attribution License which permits any use, distribution, and reproduction in any medium, provided the original author(s) and source are credited. 